\title{
Remission of Pseudotumor Cerebri Syndrome after Initiating Pregnancy and Menarche: The Puzzling Role of Female Sex Hormones
}

\author{
Valeria Dipasquale ${ }^{1}$ Anna Claudia Romeo ${ }^{1}$ Rosa Morabito ${ }^{2}$ Dominique De Vivo ${ }^{1}$ Emanuele David ${ }^{2,3}$ \\ Daniela Impollonia ${ }^{2}$ Giovanni Stroscio ${ }^{2}$ Francesca Granata ${ }^{2}$ Antonio Simone Laganà 4
}

1 Department of Pediatrics, University of Messina, Messina, Italy

2 Department of Radiology, University of Messina, Messina, Italy

${ }^{3}$ Department of Radiology, Anatomopathology and Oncology, Sapienza University of Rome, Italy

${ }^{4}$ Department of Pediatric, Gynecological, Microbiological and

Biomedical Sciences, University of Messina, Messina, Italy

\begin{abstract}
Address for correspondence Valeria Dipasquale, MD, Department of Pediatrics, University of Messina, Via Consolare Valeria, 198125 Messina, Italy (e-mail: dipasquale.valeria@libero.it).
\end{abstract}

\begin{abstract}
Keywords

- pseudotumor cerebri syndrome

- idiopathic intracranial hypertension

- female gender

- sex hormones

- pregnancy

- menarche

Pseudotumor cerebri syndrome (PTCS) is characterized by increased intracranial pressure with normal cerebrospinal fluid components and no detectable anomalies of the brain parenchyma on magnetic resonance imaging. Presenting signs and symptoms are heterogeneous, but they commonly include headache, visual disturbances (i.e., vision loss and/or double vision), and papilledema. The etiology is uncertain, but several underlying risk factors associated with PTCS have been identified, including obesity, endocrine abnormalities (e.g., hyperaldosteronism, Cushing syndrome, hyperandrogenism, and Addison disease), medications (e.g., tetracycline, recombinant growth hormone therapy), and viral infections (e.g., chickenpox). Patients with postpubertal PTCS are more typically females in their reproductive age, thereby making crucial (although not fully understood) the role of female hormones in the pathophysiology of this condition. We describe two female patients with PTCS who were followed up at our institution. They experienced prompt (and full) remission of headache and visual disturbances during the first trimester of pregnancy and within the first 2 months after the onset of menarche (i.e., at 20 and 11 years of age), respectively. We reviewed the literature searching for similar cases and hypothesized on the pathophysiologic (and still unclear) involvement of female sex hormones in regulating intracranial pressure, making some patients prone to PTCS.
\end{abstract}

\section{Introduction}

Pseudotumor cerebri syndrome (PTCS) is a condition that encompasses both primary (idiopathic) and secondary causes of intracranial hypertension (e.g., medical conditions, venous sinus thrombosis, medications). Based on new criteria, idiopathic intracranial hypertension is now considered not synonymous with PTCS but rather a subset within primary PTCS. ${ }^{1}$
PTCS is a neurologic disorder of unclear etiology, characterized by increased intracranial pressure (ICP) without any radiographic evidence of abnormal brain parenchyma, and with normal chemical and cytologic cerebrospinal fluid (CSF) composition. ${ }^{1}$ Even though occurring in both sexes and all age groups, the postpubertal disease typically affects overweight women of childbearing age. ${ }^{2}$ Notably, the overall incidence of PTCS is estimated to be 0.9 per 100,000 but rises to 19 per received

October 22, 2014 accepted after revision January 18, 2015
Issue Theme Pediatric Pseudotumor Cerebri Syndrome; Guest Editors: Vincenzo Salpietro, MD, Martino Ruggieri, PhD, and Conrad E. Johanson, PhD
Copyright ๑ 2015 Georg Thieme Verlag KG Stuttgart · New York

DOI http://dx.doi.org/ 10.1055/s-0035-1555148. ISSN $1304-2580$. 
100,000 in overweight women. ${ }^{2}$ According to recently proposed diagnostic criteria, a definitive diagnosis of PTCS requires papilledema, normal neurology (other than cranial nerve abnormalities), and CSF composition, and elevated lumbar puncture (LP) opening pressure $\left(\geq 28 \mathrm{cmH}_{2} \mathrm{O}\right.$ in children or $\geq 25 \mathrm{cmH}_{2} \mathrm{O}$ in adults or in unsedated nonobese children). ${ }^{1}$

PTCS patients typically recover with appropriate medical and/or surgical treatments, but up to $10 \%$ of the affected children may have irreversible visual loss, making the timely diagnosis and treatment of this condition an absolute priority in the pediatric age group. ${ }^{3}$ Although the pathophysiology of PTCS is still unclear, many potential risk factors associated with it have been identified, including endocrinopathies, infections, hematologic disorders, and medications ${ }^{3-5}$; unifying effects of many of these factors on increased ICP have been recently proposed. ${ }^{6}$

We hereby report two PTCS female patients who were treated and followed up at our institution. They experienced complete remission of the clinical symptoms, as evidenced by funduscopic findings during the first trimester of pregnancy and within the first 2 months of menarche onset (i.e., at 21 and 11 years of age, respectively). On the basis of previous literature reports and recent research perspectives, we now consider the potential pathophysiologic role of female sex hormones in PTCS.

\section{Case Reports}

\section{Case 1}

The first patient was initially admitted to Messina University hospital at 9 years of age, because of moderate to severe headache that was refractory to the common anti-inflammatory medications. On admission, a grade III papilledema was bilaterally noted through the funduscopic examination. Magnetic resonance imaging (MRI) and magnetic resonance venography (MRV) of the brain yielded normal results. Pelvic ultrasound scan measured uterine length, uterine area, uterine volume, and ovarian volume that were all in line (at that time) with a prepubertal developmental stage. Because of the persistence of severe headache and the finding of papilledema we performed LP and the opening CSF pressure was found to be elevated $\left(+270 \mathrm{~mm} \mathrm{H}_{2} \mathrm{O}\right)$.

She was therefore diagnosed with PTCS and put on acetazolamide $(20 \mathrm{mg} / \mathrm{kg} /$ day $)$ for 4 months, with only limited benefits. The girl complained of persistent moderate to severe headache episodes and was then switched to topiramate (15 $\mathrm{mg} / \mathrm{kg} /$ day) with a partial clinical response, but she continued to suffer generalized head pain and frequent attacks of diplopia in the months ahead. Shortly after the onset of menarche (i.e., at 10 years and 8 months), she reported to have no more episodes of headache or diplopia, and all the general conditions were slightly improved. She was then readmitted to our department at 11 years and 5 months of age. The neurologic examination was normal and the ophthalmologic diagnostic workup revealed a complete remission of papilledema. She did not need any further treatment in the months/years ahead and the follow-up ophthalmologic investigations confirmed the absence of pathologic findings in the funduscopic examination. At 15 years of age, her general health is fairly good and no any further neurologic and/or visual issues have been reported to us.

\section{Case 2}

The second girl was initially admitted to our Institution at 16 years of age because of visual loss and severe headache. She was overweight (body mass index $>90$ th percentile) and her neurologic examination showed a sixth cranial nerve palsy. The funduscopic examination showed a bilateral papilledema (grade II) and brain MRI revealed an increased tortuosity of optic nerve. She underwent LP that showed elevated CSF pressure $\left(+410 \mathrm{~mm} \mathrm{H}_{2} \mathrm{O}\right)$, confirming the diagnosis of PTCS. She started on acetazolamide $(20 \mathrm{mg} / \mathrm{kg} /$ day $)$ with partial clinical response, but she continued to complain from time to time about episodes of headache and visual disturbances.

At 18 years of age, because of the slight worsening of her clinical picture, she underwent several follow-up examinations, including funduscopy, which showed an aggravation of the papilledema (i.e., grade III). Additionally, MRI findings depicted an empty sella (-Fig. 1). Further clinical symptoms (e.g., tinnitus, double vision) also appeared in the months ahead, and despite being under combined diuretic treatment with acetazolamide $(20 \mathrm{mg} / \mathrm{kg} /$ day $)$ and furosemide $(2 \mathrm{mg} /$ $\mathrm{kg}$ /day), she continued to have headache with only limited periods free of symptoms. At 20 years of age, the woman got pregnant and, during the first trimester of gestation, she noticed a complete disappearance of headache, visual disturbances, and tinnitus. We therefore again evaluated her at our clinic and documented a quite complete resolution of papilledema at the funduscopic examination; the brain alterations previously revealed by MRI (i.e., empty sella) were also reduced. At 23 years now, the PTCS-related clinical manifestations are greatly improved after the gestation. She now occasionally complains about episodes of headache, lasting from minutes to hours; however, she has not needed any diuretic or other treatment over the past 3 years.

\section{Discussion}

PTCS pathophysiology is still poorly understood; however, the main pathologic feature of this intriguing condition is represented by elevated ICP. The skull being an enclosed compartment, in the absence of any intracranial structural anomalies, an increase in ICP may occur as the result of either increased CSF production at the choroid plexus level and/or reduced CSF outflow in the arachnoid exit sites and the cerebral venous system. ${ }^{3,6}$ We know from many clinical studies and case series that pediatric PTCS can occur in association with a broad variety of conditions, especially obesity and endocrinopathies (e.g., adrenal insufficiency, Cushing syndrome, hyperandrogenism, and hyperaldosteronism). ${ }^{4-6}$ Although prepubertal PTCS can occur in both sexes and ages, postpubertal PTCS is known to occur in women of childbearing age in the majority of cases. ${ }^{2}$ 

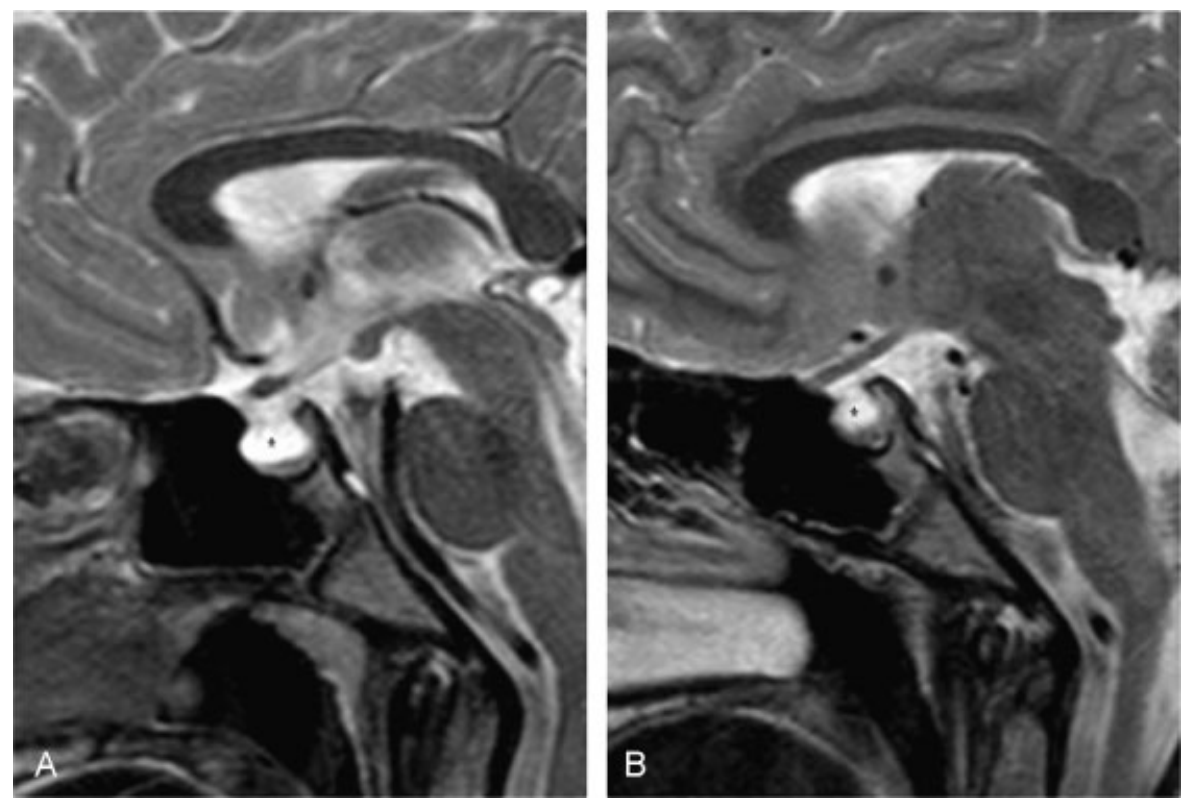

Fig. 1 (A, B) Sagittal fast-spin echo T2-weighted images show a partial empty sella (asterisks).

All this suggests that endocrine and/or metabolic derangements play an important role in initiating the rise of ICP, eventually resulting in PTCS. Recently, some studies suggested that fluid and electrolyte balance in choroid plexus epithelium (CPE), the site of CSF production, seem to be controlled by a complex interaction of metabolic and hormonal factors that likely act to regulate CSF volume and pressure. ${ }^{7,8}$ Notably, it has been previously proposed that an estrogenic gynecoid (pear-shaped) fat distribution make some women prone to develop PTCS. ${ }^{9}$ Adipose tissue contains aromatase, which may be a link between obesity and PTCS. Aromatase, producing estrogens from plasma androstenedione, is more prevalent in the buttock regions (i.e., female distribution) than in abdominal (i.e., visceral) fat. ${ }^{10}$

Polycystic ovary syndrome (PCOS) is also known to occur more frequently in the PTCS population and it is typically associated with high levels of androgen. It is unlikely that the androgens themselves predispose to PTCS, but there is enzymatic conversion to estrogens peripherally through aromatase that may be a significant factor. ${ }^{9}$ Interestingly, the reports of the onset of PTCS in postmenopausal women following the initiation of hormone replacement therapy further support the notion of an estrogen involvement in the pathophysiology of this condition. ${ }^{9}$ A recent study proposed a theory unifying the various neuroendocrine effects on the mineralocorticoid receptor (MR) pathway to explain a possible mechanism for the increased CSF production and pressure in PTCS. ${ }^{8}$ The MRs are largely expressed in the $\mathrm{CPE}$, which are putatively crucial in the regulation of CSF production. ${ }^{10}$

In a manner analogous to actions at the kidney, aldosterone and other adrenal hormones with a similar affinity for MRs (i.e., cortisol and progesterone) appear to enhance the activities of CPE sodium channels and $\mathrm{Na}^{+} / \mathrm{K}^{+}$-ATPase transporters (Na pumps), thereby increasing ventricular CSF sodium concentrations. These accelerated transport phenomena in CPE would create an osmotic gradient to augment the water flux underlying CSF production. ${ }^{11}$ CSF cortisol levels are regulated by the 11-hydroxysteroid dehydrogenase type 1 (HSD1) enzyme that is abundant in the CPE and converts inactive cortisone to cortisol, the latter acting with high affinity (i.e., similarly to aldosterone) at the $\operatorname{MR}(s)$ in the CPE. A pathophysiologic link between the HSD1 enzyme activity and predisposition to PTCS has been suggested by some authors. ${ }^{6-9}$

This neuroendocrine perspective would also justify the observation that PCTS can be sometimes triggered and/or worsened by pregnancy, ${ }^{12}$ as it is known that during the first phases of gestation a concomitant elevation of progesterone and aldosterone plasma concentrations occurs. ${ }^{6}$ Conversely, to the best of our knowledge, an improvement in clinical symptoms related to PTCS during pregnancy has been not reported so far in the medical literature. Notably, the role of the HSD1 system in the dynamics of CSF production and absorption is still unclear, but an integrated hormonal mechanism has been hypothesized by Sheldon et al. ${ }^{13}$ Accordingly, this would tentatively explain the occurrence of PTCS in the context of adrenal insufficiency (e.g., after resection of the pituitary tumors for Cushing disease), widely reported in the literature.

Sheldon et $\mathrm{al}^{13}$ suggested that proportional upregulation of HSD1 in CPE is a compensatory measure to limit existing plasma cortisol deficiencies. Through the local MR stimulation in CPE and possible links to downstream effects on sodium-actuated transport mechanisms, any sudden fall in cortisol level in blood might be counterbalanced by greater availability of cortisol within the CPE via this upregulated HSD1 mechanism. This could result in raised ICP and PTCS.

In line with the suggestions, we propose that activation of the MR pathway in the context of the hormonal changes typical of both menarche and gestation (i.e., elevation of 
estrogen and progesterone levels) may conversely have a role in the downregulation of the HSD1 enzyme, limiting the availability of active cortisol at CPE level and resulting in lowering ICP (and the remission of PTCS-related signs and symptoms) in some cases. Notably, besides our case 1 , other cases of PTCS adolescents who experienced a remission of symptoms after menarche have been reported in the medical literature. ${ }^{14,15}$ This further supports the possible (additional) action of female sex hormones changes in downregulating ICP. Further studies should be done in the future to fully understand the central puzzling role of female sex hormones in the pathophysiology of PTCS.

\section{References}

1 Friedman DI, Liu GT, Digre KB. Revised diagnostic criteria for the pseudotumor cerebri syndrome in adults and children. Neurology 2013;81(13):1159-1165

2 Radhakrishnan K, Ahlskog JE, Cross SA, Kurland LT, O'Fallon WM. Idiopathic intracranial hypertension (pseudotumor cerebri). Descriptive epidemiology in Rochester, Minn, 1976 to 1990. Arch Neurol 1993;50(1):78-80

3 Per H, Canpolat M, Gümüş $H$, et al. Clinical spectrum of the pseudotumor cerebri in children: etiological, clinical features, treatment and prognosis. Brain Dev 2013;35(6):561-568

4 Salpietro V, Ruggieri M, Sancetta F, et al. New insights on the relationship between pseudotumor cerebri and secondary hyperaldosteronism in children. J Hypertens 2012;30(3):629-630
5 Salpietro V, Chimenz R, Arrigo T, Ruggieri M. Pediatric idiopathic intracranial hypertension and extreme childhood obesity: a role for weight gain. J Pediatr 2013;162(5):1084

6 Salpietro V, Polizzi A, Bertè LF, et al. Idiopathic intracranial hypertension: a unifying neuroendocrine hypothesis through the adrenal-brain axis. Neuroendocrinol Lett 2012;33(6): 569-573

7 Khan MU, Khalid H, Salpietro V, Weber KT. Idiopathic intracranial hypertension associated with either primary or secondary aldosteronism. Am J Med Sci 2013;346(3):194-198

8 Salpietro V, Mankad K, Kinali M, et al. Pediatric idiopathic intracranial hypertension and the underlying endocrine-metabolic dysfunction: a pilot study. J Pediatr Endocrinol Metab 2014; 27(1-2):107-115

9 Andrews LE, Liu GT, Ko MW. Idiopathic intracranial hypertension and obesity. Horm Res Paediatr 2014;81(4):217-225

10 Salpietro V, Ruggieri M. Pseudotumor cerebri pathophysiology: the likely role of aldosterone. Headache 2014;54(7):1229

11 Salpietro V, Polizzi A, Di Rosa G, et al. Adrenal disorders and the paediatric brain: pathophysiological considerations and clinical implications. Int J Endocrinol 2014;2014:282489

12 Shehata HA, Okosun H. Neurological disorders in pregnancy. Curr Opin Obstet Gynecol 2004;16(2):117-122

13 Sheldon CA, Kwon YJ, Liu GT, McCormack SE. An integrated mechanism of pediatric pseudotumor cerebri syndrome: evidence of bioenergetic and hormonal regulation of cerebrospinal fluid dynamics. Pediatr Res 2015;77(2):282-289

14 Johnston I. The historical development of the pseudotumor concept. Neurosurg Focus 2001;11(2):E2

15 Tessler Z, Biedner B, Yassur Y. Benign intracranial hypertension in menarche. Compr Ther 1985;11(5):16-18 